\title{
Andreev reflections at interfaces between delta-doped GaAs and superconducting Al films
}

Taboryski, Rafael Jozef; Clausen, Thomas; Hansen, Jørn Bindslev; Skov, Johannes; Kutchinsky, Jonatan; Sørensen, Claus Birger; Lindelof, Poul Erik

Published in:

Applied Physics Letters

Link to article, DOI:

10.1063/1.117796

Publication date:

1996

Document Version

Publisher's PDF, also known as Version of record

Link back to DTU Orbit

Citation $(A P A)$ :

Taboryski, R. J., Clausen, T., Hansen, J. B., Skov, J., Kutchinsky, J., Sørensen, C. B., \& Lindelof, P. E. (1996). Andreev reflections at interfaces between delta-doped GaAs and superconducting Al films. Applied Physics Letters, 69(5), 656-658. https://doi.org/10.1063/1.117796

\section{General rights}

Copyright and moral rights for the publications made accessible in the public portal are retained by the authors and/or other copyright owners and it is a condition of accessing publications that users recognise and abide by the legal requirements associated with these rights.

- Users may download and print one copy of any publication from the public portal for the purpose of private study or research.

- You may not further distribute the material or use it for any profit-making activity or commercial gain

- You may freely distribute the URL identifying the publication in the public portal 


\title{
Andreev reflections at interfaces between $\delta$-doped GaAs and superconducting Al films
}

\author{
R. Taboryski, ${ }^{\text {a) }}$ T. Clausen, J. Bindslev Hansen, and J. L. Skov \\ Department of Physics, The Technical University of Denmark, Building 309, DK-2800 Lyngby, Denmark \\ J. Kutchinsky, C. B. Sørensen, and P. E. Lindelof \\ Niels Bohr Institute, University of Copenhagen, Universitetsparken 5, DK-2100 Copenhagen $\mathscr{D}$, Denmark
}

(Received 15 February 1996; accepted for publication 18 May 1996)

\begin{abstract}
By placing several Si $\delta$-doped layers close to the surface of a GaAs molecular beam epitaxy-grown crystal, we achieve a compensation of the Schottky barrier and obtain a good Ohmic contact between an in situ deposited (without breaking the vacuum) Al metallization layer and a highly modulation doped $\left(n^{++}\right)$conduction layer embedded below the $\delta$-doped layers in the GaAs crystal. When cooled to below the critical temperature $(\approx 1.2 \mathrm{~K})$ of $\mathrm{Al}$, superconductivity is induced in the conductive layer of the semiconductor. We have studied the current voltage $(I-V)$ characteristics in a planar geometry where the $\mathrm{Al}$ has been removed in a thin stripe. We find a manifestation of the superconducting energy gap and a rich fine structure at injection energies both below and above the gap. (C) 1996 American Institute of Physics. [S0003-6951(96)00231-8]
\end{abstract}

The study of superconductor-semiconductor (S-Sm) junctions is a field of increasing interest. For applications, the main effort has been invested in making a three-terminal device, where the field effect in the semiconductor can be used to control a supercurrent between the closely placed superconducting contacts. ${ }^{1}$ A supercurrent can flow, if the distance between the superconductors is comparable to the coherence length $\xi$ in the semiconductor, and if the S-Sm interfaces are highly transmissive. More fundamental aspects dealing with the basic understanding of the induction of superconductivity in mesoscopic (phase-coherent) normal conductors have also gained increased interest. ${ }^{2,3}$ At a normalsuperconductor $(\mathrm{N}-\mathrm{S})$ interface the well known proximity effect can, thus, be explained on a microscopic level in terms of the Andreev reflection, where an electron in the normal conductor can be transmitted as a Cooper pair into the superconductor if a hole is retroreflected along the time-reversed path of the electron.

The key parameter in the description of Andreev reflections is the dimensionless parameter $Z{ }^{4}$, which enters the expression for the normal resistance of a N-S interface $R_{N}$ $=R_{0}\left(1+Z^{2}\right) \cdot R_{0}$ is the barrierless resistance, whereas $Z$ is a measure of the effective interface barrier height. In a model system with a $\delta$-function barrier $Z$ is given by $Z$ $=\sqrt{Z_{0}+(1-r)^{2} / 4 r}$, where $Z_{0}=H / \hbar v_{F}^{\mathrm{sm}}$ and $r=v_{F}^{\mathrm{sm}} / v_{F}^{\mathrm{sp}}$. Here $H$ is the strength of the $\delta$-function barrier and $v_{F}^{\mathrm{sm}}$, $v_{F}^{\text {sp }}$ are the Fermi velocities in the normal metal and the superconductor, respectively. The contributions to $Z$, thus, come from an interface energy barrier and a Fermi velocity mismatch between the two materials. The lower the $Z$ the higher is the probability for Andreev reflection processes at the $\mathrm{S}-\mathrm{N}$ or $\mathrm{S}-\mathrm{Sm}$ interfaces. Another important quantity is the coherence length in the normal metal (or degenerated semiconductor). This length determines the length scale over which electrons and Andreev reflected holes can maintain their phase coherence. In the dirty limit where the electronic mean free path $l$ is much shorter than the coherence length,

${ }^{a)}$ Electronic mail: rjt@mips.fjg.dtu.dk $\xi=\sqrt{\hbar D / 2 \pi k_{B} T}$, where $D=\frac{1}{3} v_{F}^{\mathrm{sm}} l$ is the diffusion constant for diffusion in three-dimensions.

The above definitions of the important quantities draw the attention to the choice of materials. III-V semiconductors have played a key role, with highly doped InAs as the preferred material due to its ability to form very low interface barriers with most metals deposited on the surface. $p$-type InAs forms a two-dimensional (2D) electron gas inversion layer at the surface but, unfortunately, with a rather low mobility. More advanced materials such as InAs-AlSb quantum wells were used by Nguyen et al. ${ }^{5}$ Here a planar structure with a 2D carrier density of roughly $10^{12} \mathrm{~cm}^{-2}$ and mobilities of the order of $10 \mathrm{~m}^{2} / \mathrm{V} \mathrm{s}$ (corresponding to mean free paths of $1.3 \mu \mathrm{m}$ ) was successfully used to obtain $\mathrm{S}-\mathrm{Sm}-\mathrm{S}$ structures exhibiting well pronounced supercurrent. Kleinsasser et al. ${ }^{6}$ have employed backgated $n$-type $\mathrm{In}_{0.47} \mathrm{Ga}_{0.53} \mathrm{As}$ grown on $p$-type InP substrates to produce three-terminal Josephson field effect transistor devices. In both these works a clear excess current was observed, indicating $Z$ factors around 1 or below. Another approach is the use of annealed $\mathrm{Ti} / \mathrm{Sn}$ contacts to a GaAs/AlGaAs heterostructure containing a $2 \mathrm{D}$ electron gas buried below the surface. $^{7}$

All the above works, however, rely on rather involved processing procedures. To obtain a high interface transmissivity and a long coherence length, one needs a high doping level and a high carrier mobility in the semiconductor channel. These two demands are not easily combined, and one often has to choose a suitable compromise. In this Letter we report a new and very simple method for making planar $\mathrm{S}-\mathrm{Sm}-\mathrm{S}$ structures with very high contact transmissivities and reasonably long coherence lengths. From a technological point of view GaAs is the most studied among the III-V materials, and many experimental groups have access to molecular beam epitaxy (MBE) systems with $\mathrm{Ga}, \mathrm{As}, \mathrm{Al}$, and $\mathrm{Si}$ sources.

Our samples consisted of $200 \mathrm{~nm}$ GaAs grown in a MBE chamber on an undoped GaAs substrate. The $200 \mathrm{~nm}$ were doped with $\mathrm{Si}$ to $4.4 \times 10^{18} \mathrm{~cm}^{-3}$ and capped with 5 


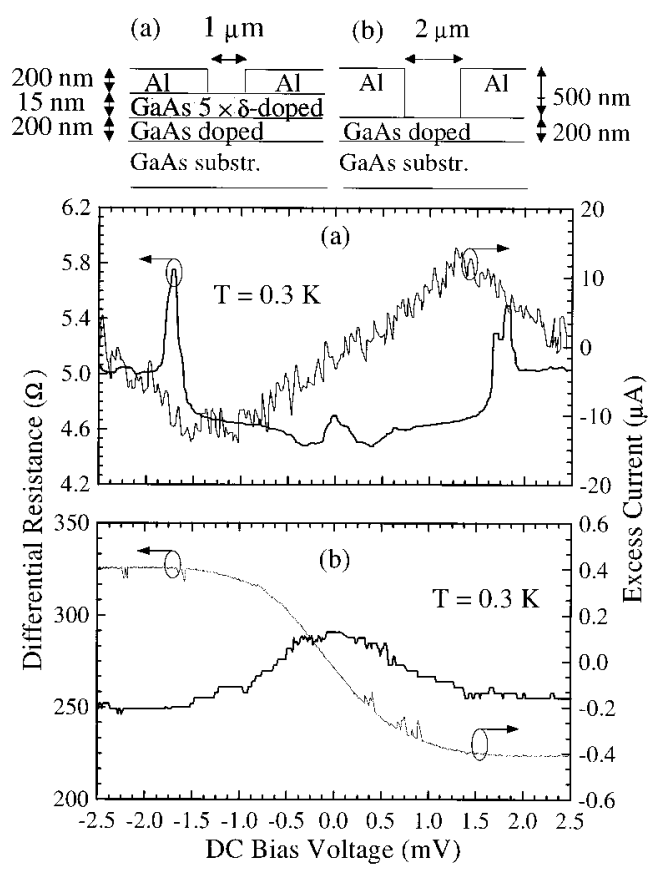

FIG. 1. (a) The differential resistance and the excess current for one of our samples with $\delta$ doping. At the sharp peaks in differential resistance (at \pm 1.8 $\mathrm{mV}$ ), the excess current is quenched due to self-heating. (b) The same quantities measured on a sample without $\delta$ doping. We notice a much higher resistance level and a deficit current instead of an excess current. The difference in the noise level in (a) and (b) is due to slightly different experimental setups when used recording the two traces. In these plots the etched stripe had the form of a straight line across the mesa. At the top of the figure we show the corresponding cross sections of the samples.

$\delta$-doped layers separated by $25 \AA$ of undoped GaAs. Each of the $\delta$-doped layers contained $5 \times 10^{13} \mathrm{~cm}^{-2} \mathrm{Si}$ atoms. These layers were inserted to decrease the Schottky barrier at the subsequent $\mathrm{Sm}-\mathrm{S}$ interface formed as the structure was terminated with $200 \mathrm{~nm}$ of pure $\mathrm{Al}$, which was deposited after the substrate temperature had fallen to about $30{ }^{\circ} \mathrm{C}$ (to prevent the formation of AlAs at the interface). As seen in Fig. 1 , the insertion of the $\delta$-doped layers had a dramatic effect on the interface resistance. The contact resistivity was lowered by three orders of magnitude from about $1 \times 10^{-7} \Omega \mathrm{m}^{2}$ to about $0.5 \times 10^{-10} \Omega \mathrm{m}^{2}$. A $17 \mu \mathrm{m}$ wide mesa structure was etched in the $\mathrm{Al}$ and the doped GaAs layer, and $\mathrm{Ti} / \mathrm{Au}$ bonding pads were deposited. A $1 \mu \mathrm{m}$ wide line was then etched in the $\mathrm{Al}$ across the mesa using conventional electron beam lithography with poly(methylmethacrylate) resist. The $\mathrm{Al}$ was wet-etched in $\mathrm{H}_{3} \mathrm{PO}_{4}: \mathrm{H}_{2} \mathrm{O}(1: 2)$ at $50{ }^{\circ} \mathrm{C}$ for about 2 min. The two-terminal resistance was dominated by the oxide barriers between the $\mathrm{Al}$ and the Ti/Au pads, whereas the four-terminal resistance exclusively probed the $1 \mu \mathrm{m}$ long $\mathrm{Al}-\mathrm{GaAs}-\mathrm{Al}$ configuration. In order to make a systematic study of the electrical properties of the $\mathrm{S}-\mathrm{Sm}-\mathrm{S}$ interface, the line across the $17 \mu \mathrm{m}$ wide mesa was etched in meander patterns of different total lengths as exemplified in the inset of Fig. 3. The mobility of the doped GaAs layer with the Al removed from the surface was $0.132 \mathrm{~m}^{2} / \mathrm{V} \mathrm{s}$ and the carrier density $4.75 \times 10^{24} \mathrm{~m}^{-3}$. This gave a mean free path of roughly $50 \mathrm{~nm}$ and a $0.3 \mathrm{~K}$ coherence length of $250 \mathrm{~nm}$. The lowest obtainable $Z$ factor for $H=0$, i.e., assuming solely a contribution from the Fermi velocity mismatch between the

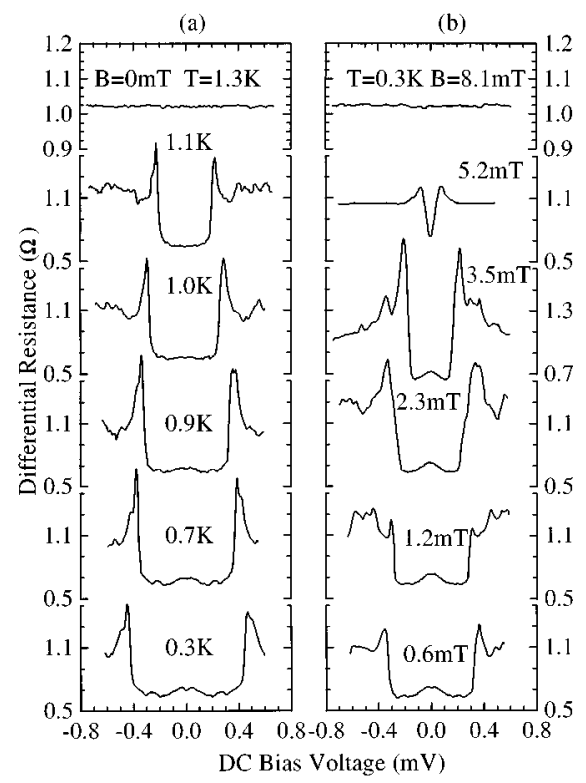

FIG. 2. (a) The resistance nonlinearity is seen to vanish as the temperature is raised to above $1.2 \mathrm{~K}$, which is the critical temperature for the Al films. We see that the zero bias anomaly is quickly quenched as the temperature is increased, while the fine structure (the subharmonic energy gap structure) is more robust. The crossover voltage at which the resistance changes from $R_{S}$ to $R_{N}$ follows roughly the temperature dependence of $\sqrt{T_{c}-T}$. (b) The nonlinearity can also be quenched by applying a magnetic field. Here the normal region, where $\mathrm{Al}$ was removed, had the form of a meandering line as shown in the inset of Fig. 3. The total length of the line was $100 \mu \mathrm{m}$.

doped GaAs layer and the Al, would be 0.39 . From our experiments we deduced $Z$ values in the range $0.7-0.9$ for the $\delta$-doped samples.

The critical temperature for our $\mathrm{Al}$ films was about 1.2 $\mathrm{K}$, and the electrical measurements were performed in a ${ }^{3} \mathrm{H}$ cryostat with a base temperature of $0.3 \mathrm{~K}$. In our experimental setup, we applied a dc voltage bias and superimposed a small sinusoidal ac modulation, that allowed us to measure the differential resistance and the $I-V$ characteristics simultaneously. The ac bias was kept sufficiently low to ensure that the measured ac voltage remained much lower than $k_{B} T / e$. Above $T_{c}$ the $I-V$ characteristics were linear. Below $T_{c}$ the characteristics became nonlinear. At very high biases the differential resistance reached the normal resistance level $R_{N}$. An excess current relative to the normal state characteristic was detected until a certain voltage $V_{c}$, where Joule heating caused a breakdown of superconductivity in the banks near the normal conductor. This breakdown resulted in an abrupt jump of the current from the excess current in the superconducting state to the normal state current. This jump gave rise to a sharp peak in the differential resistance. As seen in Fig. 1(b), samples cut from a wafer without $\delta$ doping exhibited a deficit current. Above $V_{c}$, irregularities or mesoscopic fingerprints were detected in the differential resistance on top of a constant level equal to the normal state resistance $R_{N}$. The peak heights at $\pm V_{c}$ and the mesoscopic fingerprints above $V_{c}$ and below $-V_{c}$ were not always fully symmetric. Below $V_{c}$ the differential resistance dropped to an overall level lower than $R_{N}$. This lower resistance level we denote $R_{S} . V_{c}$ is not the gap voltage $2 \Delta / e$ since it had different values for samples with different shape of the 


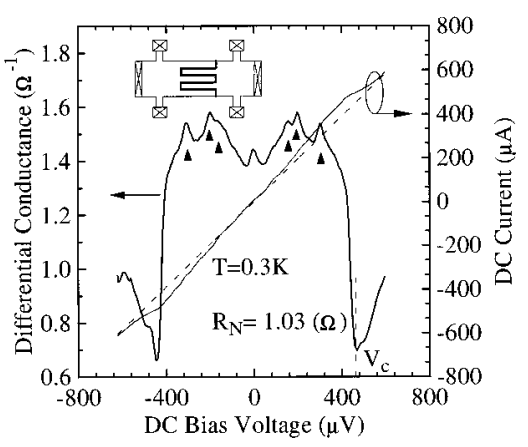

FIG. 3. The $I-V$ characteristic (thin solid line) for the same sample as shown in Fig. 2. The thick line is the differential conductance with indications of a subharmonic energy gap-structure. The dashed line indicates the $I-V$ curve corresponding to the normal resistance $R_{N}$. The inset shows the sample geometry, with a meandering line etched in the $\mathrm{Al}$ on top of the sample.

etched stripe. This is clearly seen by comparison of Figs. 1 and 2. Superimposed on $R_{S}$ was a fine structure that was symmetric around zero bias voltage. The precise shape of this fine structure was sample dependent. Around zero dc bias either a bare peak or a peak with a small dip inside it was observed. As seen in Fig. 2, the fine structure below $V_{c}$ disappeared gradually as the temperature or the magnetic field approached its critical value. The peak around zero bias is most strongly affected by the temperature and by magnetic fields. The temperature dependence of $V_{c}$ is roughly $\sqrt{T_{c}-T}$, corresponding to an energy balance where the dissipated power $P=V_{\text {appl }}^{2} / R \propto\left(T_{c}-T\right)$.

The $I-V$ characteristics can be understood if one takes into account the distribution of current (and voltage drop) away from the etched stripe, the Blonder, Tinkman, Klapwijk theory, and simple heating effects. From the excess current and the zero bias resistance level we were able to deduce $Z$ values in the range $0.7-0.9$. In a planar geometry the current density $J(x)$ through each $\mathrm{S}-\mathrm{Sm}$ interface will be a function of the distance from the edge. Given an applied potential $V_{\text {appl }}$ between the two Al banks, it can easily be shown, that the distributed voltage drop is given by

$$
\frac{d^{2} V}{d x^{2}}=-\frac{\rho_{\mathrm{sm}}}{d} J(x),
$$

where $\rho_{\text {sm }}$ is the resistivity of the semiconductor and $d$ is the thickness of the conducting semiconductor layer. The total current is given by

$$
I=w \int_{0}^{\infty} J(x) d x,
$$

where $w$ is the width of the $\mathrm{S}-\mathrm{Sm}$ interface. If the two equations above are solved in the normal state with suitable boundary conditions and $J(V(x))=V / \rho_{N}$ one gets for the normal resistance

$$
R_{N} \equiv V_{\mathrm{appl}} / I=2 \frac{\rho_{N}}{l_{N} w}+R_{\mathrm{sm}},
$$

where $R_{\mathrm{sm}}=\rho_{\mathrm{sm}} L / d w$ is the resistance of the piece of semiconductor of length $L$ between the superconducting banks, while $\rho_{N}$ is the normal state contact resistivity with dimension $\left[\Omega \mathrm{m}^{2}\right] . l_{N}=\sqrt{\rho_{N} d / \rho_{\mathrm{sm}}}$, is the decay length for $V(x)$ across each of the $\mathrm{S}-\mathrm{Sm}$ interfaces away from the etched stripe. For samples cut from the $\delta$-doped wafer $l_{N}$ was of the order of $0.5 \mu \mathrm{m}$, while for solely modulation doped samples it was roughly $60 \mu \mathrm{m}$. In the superconducting state, the energy gap will, thus, appear at voltages $V_{\text {appl }}=2 \Delta / e+R_{\mathrm{sm}} I$.

In Fig. 3 we show the $d I / d V-V$ and $I-V$ curves for a typical sample. In Fig. 3 we have indicated the positions of $\pm 2 \Delta e, \pm \Delta / e$, and $\pm 2 \Delta / 3 e$. We interpret the observed structure as subharmonic energy gap structure originating from multiple Andreev scattering across the S-Sm-S junction. ${ }^{8,9}$ This structure can persist even to $T=0$ with unaltered amplitude. The value of $\Delta$ is, however, subject to a voltage dependence due to the self-heating effect. The dip at zero bias was not present in all our samples and is, presumably, a precursor to a supercurrent, or the so-called zero bias excess conductance observed by many workers. ${ }^{10}$

In conclusion, we have demonstrated a new and very simple technique to make $\mathrm{S}-\mathrm{Sm}-\mathrm{S}$ structures with high interface transmissivities. In our samples we have observed high bias excess current and subharmonic energy gap structure. Finally, we have demonstrated how to interpret data taken in a planar geometry.

This work was supported by the Danish Technical Research Council. We also thank CNAST for support and the III-V Nanolab at the Niels Bohr Institute for providing us with the processing facilities.

${ }^{1}$ See, for example, A. W. Kleinsasser, IEEE Trans. Appl. Supercond. 3, 1968 (1993).

${ }^{2}$ H. Takayanagi, J. Bindslev Hansen, and J. Nitta, Phys. Rev. Lett. 74, 162, 166 (1995).

${ }^{3}$ A. Dimoulas, J. P. Heida, B. J. v. Wees, T. M. Klapwijk, W. v. d. Graaf, and G. Borghs, Phys. Rev. Lett. 74, 602 (1995).

${ }^{4}$ G. E. Blonder and M. Tinkham, Phys. Rev. B 27, 112 (1983); G. E. Blonder, M. Tinkham, and T. M. Klapwijk, ibid. 25, 4515 (1982).

${ }^{5}$ Chanh Nguyen, J. Werking, H. Kroemer, and E. L. Hu, Appl. Phys. Lett. 57, 87 (1990).

${ }^{6}$ A. W. Kleinsasser, T. N. Jackson, D. McInturff, F. Rammo, G. D. Pettit, and J. M. Woodall, Appl. Phys. Lett. 57, 1811 (1990).

${ }^{7}$ J. R. Gao, J. P. Heida, B. J. V. Wees, S. Bakker, T. M. Klapwijk, and B. W. Alphenaar, Appl. Phys. Lett. 63, 334 (1993); A. M. Marsh, D. A. Williams, and H. Ahmed, Phys. Rev. B 50, 8118 (1994).

${ }^{8}$ K. Flensberg, J. Bindslev Hansen, and M. Ocatavio, Phys. Rev. B 38, 8708 (1988)

${ }^{9}$ M. Octavio, M. Tinkham, G. E. Blonder, and T. M. Klapwijk, Phys. Rev. B 27, 6739 (1983).

${ }^{10}$ A. Kastalsky, A. W. Kleinsasser, L. H. Greene, R. Bhat, F. P. Milliken, and J. P. Harbison, Phys. Rev. Lett. 67, 3026 (1991); Chanh Nguyen, H. Kroemer, and E. L. Hu, ibid. 69, 2847 (1992); B. J. van Wees, P. Magnee, and T. M. Klapwijk, ibid. 69, 510 (1992). 